\title{
Join spaces, soft join spaces and lattices
}

\section{Violeta Leoreanu-Fotea, Bijan Davvaz, Feng Feng and Codrina Chiper}

In the honour of Professor Mirela Ştefănescu

\begin{abstract}
The aim of this paper is to initiate and investigate new (soft) hyperstructures, particularly (soft) join spaces, using important classes of lattices: modular and distributive. They are used in order to study (soft) hyperstructures constructed on the set of all convex sublattices of a lattice.
\end{abstract}

\section{Introduction}

There are several theories, such as probabilities, fuzzy sets, rough sets, vague sets, interval mathematics, which can be considered as mathematical tools for dealing with uncertainties. In [24], Molodtsov pointed out the difficulties of these theories, that can be due to the inadequacy of the parametrization tool of each theory and he introduced a new tool, called soft set, in order to deal with uncertainties, which is free from the difficulties of the above mentioned theories. Several applications of soft sets have been established for instance in decision making problem $[22,3,12,9]$. To address decision making problems based on fuzzy soft sets, Feng et al. introduced the concept of level soft sets of fuzzy soft sets and initiated an adjustable decision making scheme using fuzzy soft sets [10]. It is also interesting to see that soft sets are closely related to many other soft computing models such as rough sets and fuzzy

Key Words: Join space; soft join space; soft hypergroup; lattice.

2010 Mathematics Subject Classification: Primary 20N20

Received: November 2013

Revised: January 2014

Accepted: January 2014 
sets. Using soft sets as the granulation structures, Feng et al. [11] defined soft approximation spaces, soft rough approximations and soft rough sets, which are generalizations of Pawlak's rough set model based on soft sets and in some cases might provide better approximations than classical rough sets.

On the other hand, hypergroups were introduced in 1934 at the VIII-th Congress of Scandinavian Mathematicians by a French mathematician Marty [21]. Nowadays, hypergroup theory is a widely applied theory $[5,6]$.

A hypergroupoid is a nonempty set $H$ endowed with a map $\cdot: H \times H \longrightarrow$ $\mathcal{P}^{*}(H)$ called hyperoperation, where $\mathcal{P}^{*}(H)$ denotes the set of all non-empty subsets of $H$. A hypergroup is an associative hypergroupoid $(H, \cdot)$ in which $x \cdot H=H \cdot x=H$ for all $x \in H$, where for all $A, B \subseteq H$ and $x \in H$, $A \cdot B=\bigcup_{a \in A, b \in B} a \cdot b, \quad A \cdot x=A \cdot\{x\}$ and $x \cdot B=\{x\} \cdot B$. Many interesting examples of hypergroups are given in $[5,6]$.

A nonempty subset $S$ of a hypergroup $(H, \cdot)$ is called a subhypergroup if $\forall x \in S, x \cdot S=S \cdot x=S$. For any $x, y$ of $H$, we denote $x / y=\{u \mid x \in u \cdot y\}$.

Prenowitz introduced a particular type of hypergroups, called join spaces, and then founded, together with Jantosciak [28], geometries on join spaces, which became a useful instrument in the study of several matters: graphs, hypergraphs, binary relations, fuzzy sets and rough sets (see [6] for details). If $\left(H_{1}, \cdot\right)$ and $\left(H_{2}, \cdot\right)$ are hypergroups, then a map $f: H_{1} \rightarrow H_{2}$ is called a homomorphism if for all $x, y$ of $H_{1}, f(x \cdot y) \subseteq f(x) \cdot f(y)$.

A commutative hypergroup $(H, \cdot)$ is called a join space if for all $x, y, z, v$ of $H$, the following implication holds: $x / y \cap z / v \neq \emptyset \Rightarrow x \cdot v \cap z \cdot y \neq \emptyset$.

On the other hand, in order to introduce the soft set notion, we consider a universe set denoted by $U$ and a set of parameters denoted by $E$. Let $\mathcal{P}(U)$ be the power set of $U$ and $A \subseteq E$.

A pair $(f, A)$ is called a soft set over $U$, where $f: A \rightarrow \mathcal{P}(U)$ is a map. Hence, a soft set over $U$ is a parameterized family of subsets of $U$. For all $a \in A$, the subset $f(a)$ can be considered as the set of $a$-approximate elements of $(f, A)$.

Thus, for a certain element $a \in A$, the subset $f(a)$ of $U$ is composed by all the elements of $U$, which correspond to the parameter $a$.

We consider now that the universe set $U$ is a hypergroup $(H, \cdot)$ and, as above, $A$ is a nonempty set and $f: A \rightarrow \mathcal{P}(H)$ be a map.

1.1. Definition [17] A pair $(f, A)$ is called a soft hypergroup over $H$ if:

$$
\forall a \in A, f(a) \neq \emptyset \Rightarrow f(a) \text { is a subhypergroup of } H \text {. }
$$

Every fuzzy subhypergroup can be interpreted as a soft hypergroup. In- 
deed, suppose that $\mu$ is a fuzzy subhypergroup of a hypergroup $(H, \circ)$ (see [6], page 212), which means that $\mu$ satisfies the following axioms:

i) $\min \{\mu(x), \mu(y)\} \leq \inf \{\mu(z): z \in x \circ y\}$ for all $x, y \in H$;

ii) for all $x, a \in H$, there exists $y \in H$ such that $x \in a \circ y$ and $\min \{\mu(a), \mu(x)\} \leq \mu(y) ;$

iii) for all $x, a \in H$, there exists $z \in H$ such that $x \in z \circ a$ and $\min \{\mu(a), \mu(x)\} \leq \mu(z)$.

If we consider the family of $\alpha$-level sets for $\mu$, given by

$$
f(\alpha)=\{x \in H: \mu(x) \geq \alpha\},
$$

where $\alpha \in[0,1]$, then for all $\alpha \in[0,1], f(\alpha)$ is a subhypergroup of $H$. Hence $(f,[0,1])$ is a soft hypergroup over $H$.

Indeed, if $\mu$ is a fuzzy subhypergroup of a hypergroup $(H, \cdot)$ (see [6], page 212) and for all $\alpha \in[0,1], f(\alpha)$ is the $\alpha$-level set for $\mu$, then $(f,[0,1])$ is a soft hypergroup over $H$.

1.2. Example If $(L, \vee, \wedge)$ is a modular lattice and we define

$$
\forall a, b \in L, a \circ b=\{x \in L: a \vee x=b \vee x=a \vee b\},
$$

then $(L, \circ)$ is a join space (see [6], page 128). For all $a \in L, I(a)=\{x \in L$ : $x \leq a\}$ is a subhypergroup of $(L, \circ)$. We define a map $f: L \rightarrow \mathcal{P}(L)$ as follows $f(a)=I(a)$ and $(f, L)$ is a soft hypergroup over $L$.

In what follows we need the following two notions:

1.3. Definition $(H, \cdot)$ be a hypergroup. A pair $(f, A)$ is called a soft join space over $H$ if:

$$
\forall a \in A, f(a) \neq \emptyset \Rightarrow f(a) \text { is a join space of } H .
$$

The soft hypergroup of Example 1.2 is a soft join space.

1.4. Definition Let $(f, A)$ be a soft hypergroup over a hypergroup $(H, \cdot)$. A soft set $(g, B)$ over $H$ is called a soft subhypergroup of $(f, A)$ if $B \subseteq A$ and for all $b \in B$, if $g(b)$ is nonempty, then it is a subhypergroup of $f(b)$. 


\section{Join spaces and soft join spaces associated with lattices}

Now, we consider the following two hyperoperations on a lattice $(L, \vee, \wedge)$ :

$x \circ y=\{z \in L: x \vee z=y \vee z=x \vee y\}, x \diamond y=\{z \in L: x \wedge y \leq z \leq x \vee y\}$.

The hyperoperation " $\circ$ " was introduced by T. Nakano [26] and analysed by St. Comer [5], J. Mittas, M. Konstantinidou [23], I. G. Rosenberg [19, 20], B. Davvaz [18] and others. The hyperoperation " $\diamond$ " was introduced by J.C. Varlet [31] and analysed by M. Konstantinidou, S. Serafimidis, Ath. Kehagias ciiteKS, KSK, SK, V. Leoreanu-Fotea, I. G. Rosenberg [19, 20], B. Davvaz [18] and others. It is frequently used in machine learning applications. St. Comer and respectively J.C. Varlet characterized modular, respectively distributive lattices, using the above hyperoperations. These characterizations are presented in [6], Chapter 4, paragraph 3, respectively paragraph 1.

\subsection{Theorem}

i) A lattice $(L, \vee, \wedge)$ is modular if and only if $(L, \circ)$ is a join space.

ii) A lattice $(L, \vee, \wedge)$ is distributive if and only if $(L, \diamond)$ is a join space.

In order to characterize some soft join spaces, we need the following notions:

2.2. Definition [27] Let $(L, \leq)$ be a lattice. A pair $(f, A)$ is called a soft lattice over $L$ if:

$$
\forall a \in A, f(a) \neq \emptyset \Rightarrow f(a) \text { is a sublattice of } L .
$$

A soft lattice $(f, A)$ is called soft modular (distributive) over a lattice $L$ if:

$\forall a \in A, f(a) \neq \emptyset \Rightarrow f(a)$ is a modular (distributive) sublattice of $L$.

A soft set $(g, B)$ over a lattice is called a soft sublattice of $(f, A)$ if $B \subseteq A$ and for all $b \in B$, if $g(b)$ is nonempty, then it is a sublattice of $f(b)$.

There are many interesting examples of soft lattices, soft distributive (modular) lattices in [27]. We give here other examples.

\subsection{Examples}

i) Let $S$ be a semigroup and $H$ any sublattice of the lattice $L$ of all fuzzy congruences on $S$ such that $f g=g f$ for all $f, g \in H$. Then $H$ is a modular lattice, see [1]. Hence, if $T$ is a cardinally equivalent set to the set of all sublattices of $L$, then $(\varphi, T)$ is a soft modular lattice over $L$, where $\varphi$ is a bijective map from $T$ to the set of all sublattices of $L$. 
ii) According to [2], if $C_{0}, C_{1}$ are chains of a modular lattice $L$, then the sublattice $L^{\prime}$ of $L$, generated by $C_{0} \cup C_{1}$ is distributive. Hence if $T=\{t\}$ is a singleton set and $\varphi: T \rightarrow\left\{L^{\prime}\right\}$, we obtain a distributive soft lattice $(\varphi, T)$ over $L$.

2.4. Corollary A soft lattice $(f, A)$ over a lattice $L$ is modular if and only if $(f, A)$ is a soft join space over the hypergroupoid $(L, \circ)$.

Proof. A soft lattice $(f, A)$ over a lattice $L$ is modular if and only if for all $a \in A$ for which $f(a) \neq \emptyset, f(a)$ is a modular sublattice of $L$. According to Theorem $2.1 \mathrm{i})$, this happens if and only if $(f(a), \circ)$ is a join space, which means that $(f, A)$ is a soft join space over the hypergroupoid $(L, \circ)$.

The following notion helps us to characterize soft subhypergroup of the associated soft join space $(f, A)$ over the hypergroupoid $(L, \circ)$.

2.5. Definition [27] Let $(f, A)$ be a soft lattice over a lattice $L$. A soft set $(g, B)$ over $L$ is called a soft ideal of $(f, A)$ if $B \subseteq A$ and for all $b \in B$, if $g(b)$ is nonempty, then it is an ideal of $f(b)$.

2.6. Theorem Let $(f, A)$ be a soft modular lattice over a lattice L. A soft set $(g, B)$ over $L$ is a soft ideal of $(f, A)$ if and only if $(g, B)$ is a soft subhypergroup of $(f, A)$, with respect to the hyperoperation " $\circ$ ".

Proof. Suppose that $(g, B)$ is a soft ideal of $(f, A)$. Then for all $b \in B$, if $g(b)$ is nonempty, then it is an ideal of $f(b)$. According to the above corollary, $(f(b), \circ)$ is a join space. Set $x, y$ be elements of $g(b)$. If $u \in x \circ y$, then $u \leq x \vee y$, and so $u \in g(b)$. Hence $g(b) \circ g(b) \subseteq g(b)$. On the other hand, for all $x, y$ elements of $g(b)$, there exists $z=x \vee y$, such that $x \in y \circ z \cap z \circ y$. Therefore, $(g(b), \circ)$ is a subhypergroup of the hypergroup $(f(b), \circ)$, which means that $(g, B)$ is a soft subhypergroup of $(f, A)$.

Conversely, suppose that $(g, B)$ is a soft subhypergroup of $(f, A)$. We check that for all $b \in B$, if $g(b)$ is nonempty, then it is an ideal of the modular lattice $f(b)$. Set $x, y$ be elements of $g(b)$. We have $x \vee y \in x \circ y \subseteq g(b)$. Moreover, if $x \in g(b)$ and $u \leq z$, where $u \in f(b)$, then $u \in z \circ z \subseteq g(b)$. Hence $g(b)$ is an ideal of $f(b)$ and so $(g, B)$ is a soft ideal of $(f, A)$.

2.7. Corollary $A$ soft lattice $(f, A)$ over a lattice $L$ is distributive if and only if $(f, A)$ is a soft join space over the hypergroupoid $(L, \diamond)$.

Proof. It follows from Theorem 2.1. ii). 
2.8. Theorem Let $(f, A)$ be a soft distributive lattice over a lattice $L$. If a soft set $(g, B)$ over $L$ is a soft ideal of $(f, A)$, then it is a soft subhypergroup of $(f, A)$, with respect to the hyperoperation " $\diamond . "$

Proof. Let $b \in B$, such that $g(b)$ is nonempty. According to the above corollary, $(f(b), \diamond)$ is a join space. Let $x, y \in g(b)$ and $z \in x \diamond y$. Hence $x \wedge y \leq z \leq x \vee y$. Since $g(b)$ is a ideal of $f(b)$, it follows that $x \vee y \in g(b)$ and then $z \in g(b)$. Thus, $x \diamond y \subseteq g(b)$. Moreover, $x \in x \diamond y \cap y \diamond x$, whence $x \in g(b) \diamond y \cap y \diamond g(b)$. Hence $g(b)=g(b) \diamond y=y \diamond g(b)$, for all $y \in g(b)$. Therefore $g(b)$ is a subhypergroup of $(f(b), \diamond)$, which means that $g(b)$ is a soft subhypergroup of the soft hypergroup $(f, A)$.

Notice that the converse is not true, as we can see from the following example:

2.9. Example Let $(L=\mathcal{P}(M), \subseteq)$ be the lattice of all parts of a nonempty set $M, A=B=\{a\}, f(a)=L$ and $g(a)=\{U \in \mathcal{P}(M) \mid C \cap D \leq U \leq C \cup D\}$, where $C, D$ are nonempty subsets of $M$, which have a nonempty intersection. Then $(g(a), \circ)$ is a subhypergroup of $(\mathcal{P}(M), \circ)$, which means that $(g, B)$ is a soft subhypergroup of $(f, A)$. On the other hand, $g(a)$ is not an ideal of $f(a)=L$. Indeed, $\emptyset \subseteq C, C \in g(a)$, but $\emptyset \notin g(a)$. This means that $(g, B)$ is not a soft ideal of $(f, A)$.

2.10. Theorem If $(f, A)$ be a soft distributive lattice over a lattice $L$ and if $(g, B)$ is a soft subhypergroup of $(f, A)$, with respect to the hyperoperation $" \diamond "$, then $(g, B)$ is a soft sublattice of $(f, A)$.

Proof. Indeed, if $b \in B$ such that $g(b)$ is nonempty. If $x, y \in g(b)$, then $x \diamond y \in g(b)$, whence for all $z \in f(b)$ such that $x \wedge y \leq z \leq x \vee y$, then $z \in g(b)$. We take $z_{1}=x \vee y$ then $z_{2}=x \wedge y$. Hence $x, y \in g(b)$ implies that $x \vee y \in g(b)$ and $x \wedge y \in g(b)$, which means that $(g, B)$ is a soft subsemilattice of $(f, A)$.

We present now some results concerning soft lattice homomorphisms.

2.11. Definition [27] Let $(f, A)$ and $(h, B)$ be two soft lattices over $L_{1}$ and $L_{2}$ respectively. Let $\varphi: L_{1} \rightarrow L_{2}$ and $\psi: A \rightarrow B$ be two maps. The pair $(\varphi, \psi)$ is called a soft lattice homomorphism if $\varphi$ is a lattice homomorphism and for all $a \in A, \varphi(f(a))=h(\psi(a))$.

If $\varphi$ is a lattice isomorphism and $\psi$ is a bijection, then $(\varphi, \psi)$ is called a soft lattice isomorphism. 
2.12. Definition [27] Let $(f, A)$ and $(h, B)$ be two soft hypergroups over the hypergroups $\left(H_{1}, \cdot\right)$ and $\left(H_{2}, \cdot\right)$ respectively. Let $\varphi: H_{1} \rightarrow H_{2}$ and $\psi: A \rightarrow B$ be two maps. The pair $(\varphi, \psi)$ is called a soft hypergroup homomorphism if $\varphi$ is a hypergroup homomorphism and for all $a \in A$, $\varphi(f(a))=h(\psi(a))$.

Notice that if $x, y \in f(a)$, then $\varphi(x), \varphi(y) \in h(\psi(a))$ according to the above definition. In other words, if $x, y$ belong to a certain subhypergroup of $\left(H_{1}, \cdot\right)$, then their images under $\varphi$ belong both to the same subhypergroup of $\left(H_{2}, \cdot\right)$.

We need to introduce the dual hyperoperation " * of " $\circ$ " on a lattice $L$, defined as follows:

$$
x * y=\{z \in L: x \wedge z=y \vee z=x \wedge y\}
$$

2.13. Theorem Using the above notations, $(\varphi, \psi)$ is a homomorphism of soft modular lattices if and only if $\varphi$ is a soft hypergroup homomorphism from $(f, A)$ to $(h, B)$, with respect to both hyperoperations " $\circ "$ and $" *$ ".

Proof. First, notice that since $(f, A)$ and $(h, B)$ are soft modular lattices, it follows that $(f, A)$ is a soft join space over both $\left(L_{1}, \circ\right)$ and $\left(L_{1}, *\right)$, while $(h, B)$ is a soft join space over both $\left(L_{2}, \circ\right)$ and $\left(L_{2}, *\right)$. We have to check that $\varphi$ is a lattice homomorphism from $L_{1}$ to $L_{2}$ if and only if $\varphi$ is both a hypergroup homomorphism from $\left(L_{1}, \circ\right)$ to $\left(L_{2}, \circ\right)$ and a hypergroup homomorphism from $\left(L_{1}, *\right)$ to $\left(L_{2}, *\right)$. Suppose that $\varphi$ is a lattice homomorphism. Since

$$
\varphi(x \circ y)=\left\{\varphi(z) \mid z \in L_{1}, z \vee x=x \vee y=y \vee z\right\},
$$

it follows that $\varphi(z) \in \varphi(x) \circ \varphi(y)$, whence $\varphi(x \circ y) \subseteq \varphi(x) \circ \varphi(y)$. Similarly, $\varphi(x \wedge y)=\varphi(x) \wedge \varphi(y)$, for all $x, y \in L_{1}$ implies that $\varphi(x * y) \subseteq \varphi(x) * \varphi(y)$. Hence $\varphi$ is both a hypergroup homomorphism from $\left(L_{1}, \circ\right)$ to $\left(L_{2}, \circ\right)$ and a hypergroup homomorphism from $\left(L_{1}, *\right)$ to $\left(L_{2}, *\right)$.

Conversely, if $u \in x \circ y$, then $\varphi(u) \in \varphi(x) \circ \varphi(y)$, that is $\varphi(u) \vee \varphi(x)=$ $\varphi(u) \vee \varphi(y)=\varphi(x) \vee \varphi(y) \geq \varphi(u)$. In particular, we take $u=x \vee y$ and so we obtain $\varphi(x) \vee \varphi(y) \geq \varphi(x \vee y)$. On the othar hand, notice that for all $u \leq x$, that is $u \in x \circ x$ we have $\varphi(u) \in \varphi(x) \circ \varphi(x)$, whence $\varphi(u) \leq \varphi(x)$. Thus, $\varphi(x) \leq \varphi(x \vee y), \varphi(y) \leq \varphi(x \vee y)$, whence $\varphi(x) \vee \varphi(y) \leq \varphi(x \vee y)$. Therefore, for all $x, y \in L_{1}, \varphi(x) \vee \varphi(y)=\varphi(x \vee y)$. Similarly, from $\varphi(x * x) \subseteq \varphi(x) * \varphi(y)$, for all $x, y \in L_{1}$, it follows that $\varphi(x) \wedge \varphi(y)=\varphi(x \wedge y)$ and so, $\varphi$ is a lattice homomorphism.

A similar result can be obtained with respect to the hyperoperation " $\diamond$ : 
2.14. Theorem $(\varphi, \psi)$ is a homomorphism of soft distributive lattices if and only if $\varphi$ is monotone and it is a soft hypergroup homomorphism from $(f, A)$ to $(h, B)$, with respect to the hyperoperation " $\diamond "$.

Proof. Notice that since $(f, A)$ and $(h, B)$ are soft distributive lattices, it follows that $(f, A),(h, B)$ are soft join spaces over $\left(L_{1}, \diamond\right)$ and $\left(L_{2}, \diamond\right)$ respectively. We have to check that $\varphi$ is a lattice homomorphism from $L_{1}$ to $L_{2}$ if and only if $\varphi$ is monotone and it is a hypergroup homomorphism from $\left(L_{1}, \diamond\right)$ to $\left(L_{2}, \diamond\right)$. Suppose that $\varphi$ is a lattice homomorphism, so it is monotone. Since

$$
\varphi(x \diamond y)=\left\{\varphi(z) \mid z \in L_{1}, x \wedge y \leq z \leq x \vee y\right\},
$$

it follows that $\varphi(z) \in \varphi(x) \diamond \varphi(y)$, whence $\varphi(x \diamond y) \subseteq \varphi(x) \diamond \varphi(y)$.

Conversely, if $u \in x \diamond y$, then $\varphi(u) \in \varphi(x) \diamond \varphi(y)$, that is $\varphi(x) \wedge \varphi(y) \leq$ $\varphi(u) \leq \varphi(x) \vee \varphi(y)$. In particular, we take $u=x \wedge y$ and $u=x \vee y$ and so we obtain $\varphi(x) \wedge \varphi(y) \leq \varphi(x \wedge y), \varphi(x \vee y) \leq \varphi(x) \vee \varphi(y)$. On the other hand, $\varphi$ is monotone and so $\varphi(x \wedge y) \leq \varphi(x) \wedge \varphi(y)$ and $\varphi(x) \vee \varphi(y) \leq \varphi(x \vee y)$. Hence $\varphi$ is a lattice homomorphism from $L_{1}$ to $L_{2}$.

\section{Join spaces associated with convex sublattices of a lat- tice}

We denote by $C S(L)$ the set of all convex sublattices of a lattice $L$. In [16] Lavanya and Parameswara Bhatta defined a partial order $\leq$ on $C S(L)$ as follows: for all $X, Y \in C S(L), X \leq Y$ if and only if

$(\forall x \in X, \exists y \in Y: x \leq y)$ and $\left(\forall y^{\prime} \in Y, \exists x^{\prime} \in X: x^{\prime} \leq y^{\prime}\right)$.

Then $(C S(L), \leq)$ is a lattice, in which

$$
\begin{gathered}
\inf \{X, Y\}=<\{x \wedge y \mid x \in X, y \in Y\}> \\
=\left\{u \in L \mid x \wedge y \leq u \leq x_{1} \wedge y_{1} \text { for some } x, x_{1} \in X \text { and } y, y_{1} \in Y\right\}, \\
\sup \{X, Y\}=<\{x \vee y \mid x \in X, y \in Y\}> \\
=\left\{u \in L \mid x \vee y \leq u \leq x_{1} \vee y_{1} \text { for some } x, x_{1} \in X \text { and } y, y_{1} \in Y\right\},
\end{gathered}
$$

where for all nonempty subset $H$ of $L,\langle H>$ denotes the convex sublattice of $L$ generated by $H$. For all $X, Y \in C S(L)$, denote

$$
X \bar{\diamond}=\{Z \in C S(L): \inf \{X, Y\} \leq Z \leq \sup \{X, Y\}\} .
$$

3.1. Theorem $(L, \diamond)$ is a join space if and only if $(C S(L), \bar{\diamond})$ is a join space. 
Proof. According to Theorem 2.1., (ii), $(L, \diamond)$ is a join space if and only if the lattice $(L, \vee, \wedge)$ is distributive. First, we check that if $(L, \vee, \wedge)$ is distributive, then the lattice $(C S(L)$, sup, inf $)$ is distributive, too.

Set $X, Y, Z \in C S(L)$. We obtain

$$
\begin{gathered}
(X \text { inf } Y) \sup Z=\left\{u \in L \mid \exists x_{i} \in X, \exists y_{i} \in Y, \exists z_{i} \in Z, i=1,2,:\right. \\
\left.t_{1} \vee z_{1} \leq u \leq t_{2} \vee z_{2}, \text { where } x_{1} \wedge y_{1} \leq t_{i} \leq x_{2} \wedge y_{2}\right\} \\
=\left\{u \in L \mid \exists x_{i} \in X, \exists y_{i} \in Y, \exists z_{i} \in Z, i \in\{1,2\}:\right. \\
\left.\left(x_{1} \wedge y_{1}\right) \vee z_{1} \leq u \leq\left(x_{2} \wedge y_{2}\right) \vee z_{2}\right\} \text { and } \\
(X \text { sup } Z) \text { inf }(Y \text { sup } Z)=\left\{v \in L \mid \exists x_{i} \in X, \exists y_{i} \in Y, \exists z_{i} \in Z, i \in\{1,2\}:\right. \\
\left.s_{1} \wedge p_{1} \leq v \leq s_{2} \wedge p_{2}, \text { where } x_{1} \vee z_{1} \leq s_{i} \leq x_{2} \vee z_{2}, y_{1} \vee z_{1} \leq p_{i} \leq y_{2} \vee z_{2}, i=1,2\right\} \\
=\left\{v \in L \mid \exists x_{i} \in X, \exists y_{i} \in Y, \exists z_{i} \in Z, i \in\{1,2\}:\right. \\
\left.\left(x_{1} \vee z_{1}\right) \wedge\left(y_{1} \vee z_{1}\right) \leq v \leq\left(x_{2} \vee z_{2}\right) \wedge\left(y_{2} \vee z_{2}\right)\right\} .
\end{gathered}
$$

Since $(L, \vee, \wedge)$ is distributive, it follows that $(C S(L), \bar{\diamond})$ is a join space. Conversely, for all $x, y, z \in L$ it is sufficient to set $X=\{x\}, Y=\{y\}, Z=\{z\}$.

Now, let $(f, A)$ be a soft join space over $(L . \diamond)$. We define $\bar{f}: A \rightarrow C S(L)$ by $\bar{f}(a)=C S(f(a))$. By the above theorem and Corollary 2.7., we obtain

3.2. Corollary $(f, A)$ is a soft join space over $(L, \diamond)$ if and only if $(\bar{f}, A)$ is a soft join space over $(C S(L), \bar{\diamond})$.

Now, for all $X, Y \in C S(L)$, denote

$$
X \bar{\circ} Y=\{Z \in C S(L): \sup \{X, Y\}=\sup \{Y, Z\}=\sup \{X, Z\}\} .
$$

3.3. Theorem $(L, \circ)$ is a join space if and only if $(C S(L), \bar{o})$ is a join space.

Proof. According to Theorem 2.1., (i), $(L, \circ)$ is a join space if and only if the lattice $(L, \vee, \wedge)$ is modular. First, we check that if $(L, \vee, \wedge)$ is modular, then the lattice $(C S(L)$, sup, inf $)$ is modular.

Set $X, Y, Z \in C S(L), X \leq Z$. It is sufficient to check that

$$
(*) \quad(X \sup Y) \inf Z \leq X \sup (Y \inf Z),
$$

which means that $\forall u \in(X \sup Y)$ inf $Z, \quad \exists v \in X \sup (Y$ inf $Z)$ such that $u \leq v$ and $\forall \bar{v} \in X \sup (Y \inf Z), \exists \bar{u} \in(X \sup Y)$ inf $Z$, such that $\bar{u} \leq \bar{v}$. 
Set $u \in(X \sup Y)$ inf $Z$. Hence, there exist $x_{i} \in X, y_{i} \in Y, z_{i} \in Z, i \in$ $\{1,2\}$, such that $t_{1} \wedge z_{1} \leq u \leq t_{2} \wedge z_{2}$, where $t_{i} \in X$ sup $Y$. Hence for $i \in\{1,2\}$, $x_{1} \vee y_{1} \leq t_{i} \leq x_{2} \vee y_{2}$. It follows that $\left(x_{1} \vee y_{1}\right) \wedge z_{1} \leq u \leq t_{2} \wedge z_{2} \leq\left(x_{2} \vee y_{2}\right) \wedge z_{2}$. Since $X \leq Z$, it follows that $\forall x \in X, \exists \bar{z} \in Z: x \leq \bar{z}$ and $\forall z \in Z, \exists \bar{x}: \bar{x} \leq z$. So, for $x_{1} \in X, \exists \bar{z}_{1} \in Z: x_{1} \leq \bar{z}_{1}$ and for $z_{1} \in Z_{1}, \exists \bar{x}_{1}: \bar{x}_{1} \leq z_{1}$. Hence $x_{1} \wedge \bar{x}_{1} \leq z_{1} \wedge \bar{z}_{1}$.

By the modularity of $L$, it follows that

$$
\left(x_{1} \vee y_{1}\right) \wedge z_{1} \geq\left[\left(x_{1} \wedge \bar{x}_{1}\right) \vee y_{1}\right] \wedge\left(z_{1} \wedge \bar{z}_{1}\right)=\left(x_{1} \wedge \bar{x}_{1}\right) \vee\left(y_{1} \wedge z_{1} \wedge \bar{z}_{1}\right) .
$$

Denote $x_{1} \wedge \bar{x}_{1}=x_{3} \in X, z_{1} \wedge \bar{z}_{1}=z_{3} \in Z$. Hence $u \geq x_{3} \vee\left(y_{1} \wedge z_{3}\right)$.

On the other hand, for $x_{2}, \exists \bar{z}_{2}: x_{2} \leq \bar{z}_{2}$ and for $z_{2}, \exists \bar{x}_{2}: \bar{x}_{2} \leq z_{2}$. Hence $x_{2} \vee \bar{x}_{2} \leq z_{2} \vee \bar{z}_{2}$. By the modularity of $L$ it follows that

$$
\left(x_{2} \vee y_{2}\right) \wedge z_{2} \leq\left[\left(x_{2} \vee \bar{x}_{2}\right) \vee y_{2}\right] \wedge\left(z_{2} \vee \bar{z}_{2}\right)=\left(x_{2} \vee \bar{x}_{2}\right) \vee\left(y_{2} \wedge\left(z_{2} \vee \bar{z}_{2}\right)\right) .
$$

Denote $x_{2} \vee \bar{x}_{2}=x_{4} \in X, \quad z_{2} \vee \bar{z}_{2}=z_{4} \in Z$. We obtain $u \leq x_{4} \vee\left(y_{2} \wedge z_{4}\right)$. Therefore, $\exists x_{3}, x_{4} \in X, \quad \exists y_{1}, y_{2} \in Y, \quad \exists z_{3}, z_{4} \in Z$ such that $x_{3} \vee\left(y_{1} \wedge z_{3}\right) \leq$ $u \leq x_{4} \vee\left(y_{2} \wedge z_{4}\right)$, whence

$$
\begin{gathered}
X \sup (Y \inf Z)=\left\{v \in L \mid \exists x_{i} \in X, \exists t_{i} \in Y \text { inf } Z:\right. \\
\left.x_{1} \vee t_{1} \leq v \leq x_{2} \vee t_{2}, \exists y_{i} \in Y, \exists z_{i} \in Z: y_{1} \wedge z_{1} \leq t_{1}, t_{2} \leq y_{2} \wedge z_{2}\right\} .
\end{gathered}
$$

Hence $x_{1} \vee\left(y_{1} \wedge z_{1}\right) \leq x_{1} \vee t_{1} \leq v \leq x_{2} \vee t_{2} \leq x_{2} \vee\left(y_{2} \wedge z_{2}\right)$, whence it follows $(*)$. Conversely, for all $x, y, z \in L$ it is sufficient to set $X=\{x\}, Y=\{y\}, Z=\{z\}$.

3.4. Corollary $(f, A)$ is a soft join space over $(L, \circ)$ if and only if $(\bar{f}, A)$ is a soft join space over $(C S(L), \overline{0})$.

Proof. It follows from Theorem 3.3 and Corollary 2.4.

\section{Conclusion}

This paper continues the study of soft hyperstructures initiated in [17], by proposing and analysing new soft hyperstructures in connection with modular and distributive lattices. The results obtained in this context are used in the study of (soft) hyperstructures obtained on the set of all convex sublattices of a lattice. 


\section{Acknowledgements}

The authors are highly grateful to the anonymous for their insightful comments and valuable suggestions. This work was partially supported by the Scientific Research Program Funded by Shaanxi Provincial Education Department (Program No. 2013JK1098).

The publication of this paper is partially supported by the grant PN-II-IDWE-2012-4-169.

\section{References}

[1] N. Ajmal, The lattice of fuzzy normal subgroups is modular, Information Sciences 83(1996), 199-209.

[2] G. Birkhoff, Lattice Theory, third ed., Colloq. Publ., 25, Amer. Math. Soc., Providence R.I., 1967.

[3] N. Çă̆man, S. Enginoğlu, Soft set theory and uni-int decision making, European Journal of Operational Research 207(2010), 848-855.

[4] P. Corsini, Prolegomena of Hypergroup Theory, second edition, (Aviani Editore, 1993).

[5] S. Comer, Multi-valued algebras and their graphical representations, Math. Comp. Sci. Dep. the Citadel. Charleston, South Carolina, 29409, July 1986.

[6] P. Corsini, V. Leoreanu, Applications of Hyperstructure Theory, (Kluwer Academic Publishers, Advances in Mathematics, no. 5, 2003).

[7] I. Cristea, Sanja Jancic-Rasovic, Composition hyperrings , Analele Stiintifice Ale Universitatii Ovidius Constanta-Seria Matematica, 21(2)(2013), 81-94.

[8] I. Cristea, Complete Hypergroups, 1-Hypergroups and Fuzzy Sets , Analele Stiintifice Ale Universitatii Ovidius Constanta-Seria Matematica, $\mathbf{1 0}(2)(2002), 25-38$.

[9] F. Feng, Y.M. Li, V. Leoreanu-Fotea, Application of level soft sets in decision making based on interval-valued fuzzy soft sets, Computers and Mathematics with Applications 60(2010), 1756-1767.

[10] F. Feng, Y.B. Jun, X.Y. Liu, L.F. Li, An adjustable approach to fuzzy soft set based decision making, Journal of Computational and Applied Mathematics 234(2010), 10-20. 
[11] F. Feng, X.Y. Liu, V. Leoreanu-Fotea, Y.B. Jun, Soft sets and soft rough sets, Information Sciences 181(2011), 1125-1137.

[12] F. Feng, Y.M. Li, N. Cagman, Generalized uni-int decision making schemes based on choice value soft sets, European Journal of Operational Research 220(2012), 162-170.

[13] M. Jafarpour, S.Sh. Mousavi, V. Leoreanu-Fotea, A class of semihypergroups connected to preordered weak Gamma-semigroups, Computers and Mathematics with Applications 62(2011), 2944-2949.

[14] Ath. Kehagias and K. Serafimidis, The L-Fuzzy Nakano Hypergroup, Information Sciences 169(2005), 305-327.

[15] Ath. Kehagias, K. Serafimidis and M. Konstantinidou, A Note on the Congruences of the Nakano Superlattice and Some Properties of the Associated Quotients, Rendiconti del Circolo Matematico del Palermo, 51(2002), 333-354.

[16] S. Lavanya, N.P. Bhatta, A new approach to the lattice of convex sublattices of a lattice, Algebra Universalis 35(1996), 63-71.

[17] V. Leoreanu-Fotea, P. Corsini, Soft hypergroups, Critical Review, Creighton University-USA 4(2010), 81-97.

[18] V. Leoreanu- Fotea, B. Davvaz, Join n-spaces and lattices, Journal of Multiple Valued Logic and Soft Computing, 15(5-6)(2009), 421-432.

[19] V. Leoreanu-Fotea, I. G. Rosenberg, Join spaces determined by lattices, Journal of Multiple Valued Logic Soft Computing, 16(1-2)(2010), 7-16.

[20] V. Leoreanu Fotea, I.G. Rosenberg, Hypergroupoids determined by lattices, European Journal of Combinatorics, 31(2010), 925-931.

[21] F. Marty, Sur une generalisation de la notion de groupe, 8th Congres Math. Scandinaves, Stockholm, 45-49, 1934.

[22] P.K. Maji, A.R. Roy, R. Biswas, An application of soft sets in a decision making problem, Computers and Mathematics with Applications 44(2002), 1077-1083.

[23] J. Mittas, M. Konstantinidou, Contributions a la theorie des treillis en liaison avec des structures hypercompositionelles y attachees, Rivista di Matematica Pura e Applicata, 14(1994), 83-114. 
[24] D. Molodtsov, Soft set theory-First results, Computers and Mathematics with Applications 37(1999), 19-31.

[25] S.Sh. Mousavi,V. Leoreanu-Fotea, M. Jafarpour, H. Babaei, Equivalence relations in semihypergroups and the corresponding quotient structures, European Journal of Combinatorics 33(2012), 463-473.

[26] T. Nakano, Rings and partly ordered systems, Math. Z. 99(1967), 355-376.

[27] K.R. Nagarajan, G. Meenambigai, An application of soft sets to lattices, Kragujevac Journal of Mathematics 35(1)(2011), 75-87.

[28] W. Prenowitz, J. Jantosciak, Join Geometries, (Springer-Verlag, UTM., 1979).

[29] K. Serafimidis and Ath. Kehagias, Some Remarks on Congruences obtained from the L-Fuzzy Nakano Hyperoperation, Information Sciences, 176(3)(2006), 301-320.

[30] M. Tarnauceanu, Actions of groups on lattices, Analele Stiintifice Ale Universitatii Ovidius Constanta-Seria Matematica, 10(1)(2002), 135-148.

[31] J.C. Varlet, Remarks on Distributive Lattices, Bull. de l 'Acad. Polonnaise des Sciences, Serie des Sciences Math., Astr. et Phys., 23(1975), 11431147.

Violeta LEOREANU-FOTEA,

Faculty of Mathematics,

"Al.I. Cuza" University,

Iaşi, Romania.

Email: leoreanu2002@yahoo.com

Bijan DAVVAZ,

Department of Mathematics,

Yazd University, Yazd, Iran

Email: davvaz@yazd.ac.ir

Feng FENG,

Department of Applied Mathematics, School of Science

Xi'an University of Posts and Telecommunications

Xi'an 710121, China Email: fengnix@hotmail.com

Codrina CHIPER,

Faculty of Mathematics,

"Al.I. Cuza" University,

Iaşi, Romania.

Email: codrina.chiper@gmail.com 